\title{
A tiny event producing an interplanetary type III burst ${ }^{\star}$
}

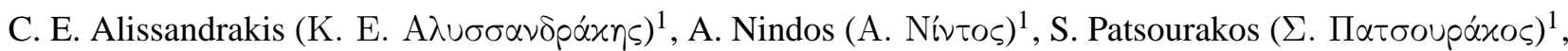

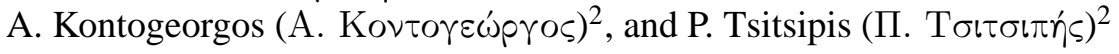 \\ 1 Department of Physics, University of Ioannina, 45110 Ioannina, Greece \\ e-mail: calissan@cc.uoi.gr \\ 2 Technological Educational Institute of Lamia, 35100 Lamia, Greece
}

Received 6 April 2015 / Accepted 28 July 2015

\begin{abstract}
Aims. We investigate the conditions under which small-scale energy release events in the low corona gave rise to strong interplanetary (IP) type III bursts.

Methods. We analyzed observations of three tiny events, detected by the Nançay Radio Heliograph (NRH), two of which produced IP type III bursts. We took advantage of the NRH positioning information and of the high cadence of AIA/SDO data to identify the associated extreme-UV (EUV) emissions. We measured positions and time profiles of the metric and EUV sources.

Results. We found that the EUV events that produced IP type III bursts were located near a coronal hole boundary, while the one that did not was located in a closed magnetic field region. In all three cases tiny flaring loops were involved, without any associated mass eruption. In the best observed case, the radio emission at the highest frequency ( $435 \mathrm{MHz}$ ) was displaced by $\sim 55^{\prime \prime}$ with respect to the small flaring loop. The metric type III emission shows a complex structure in space and in time, indicative of multiple electron beams, despite the low intensity of the events. From the combined analysis of dynamic spectra and NRH images, we derived the electron beam velocity as well as the height, ambient plasma temperature, and density at the level of formation of the $160 \mathrm{MHz}$ emission. From the analysis of the differential emission measure derived from the AIA images, we found that the first evidence of energy release was at the footpoints, and this was followed by the development of flaring loops and subsequent cooling.

Conclusions. Even small energy release events can accelerate enough electrons to give rise to powerful IP type III bursts. The proximity of the electron acceleration site to open magnetic field lines facilitates the escape of the electrons into the interplanetary space. The offset between the site of energy release and the metric type III location warrants further investigation.
\end{abstract}

Key words. Sun: radio radiation - Sun: UV radiation - Sun: flares - Sun: corona - Sun: magnetic fields

\section{Introduction}

The reconfiguration of the solar magnetic field to a lower energy state, which may be facilitated by magnetic reconnection, is the source of large amounts of energy released during solar flares. This energy leads to particle acceleration, either in the energy release region itself, or in its vicinity. Beams of energetic electrons can form either from the acceleration mechanism itself or through the propagation process. Instabilities in the beam may generate Langmuir waves that can be transformed in part into electromagnetic waves at the plasma frequency or its second harmonic via scattering off thermal ions or, more likely, coupling to low-frequency waves. This is the plasma emission mechanism; for more details, see Melrose (1980), Tsytovich (1995), and reviews by Dulk (1985), Bastian et al. (1998), Nindos et al. (2008), Melrose (2009), and Reid \& Ratcliffe (2014).

In radio dynamic spectra, the plasma emission from beams of energetic electrons usually appears as intense bands of emission drifting rapidly to low or high frequencies, depending on whether the beams travel away from or toward the Sun. Bursts drifting to lower frequencies are called type III bursts, whereas those drifting to higher frequencies are called reverse-slope bursts. Type III bursts propagate out to regions of very low density, and therefore the exciter corresponds to radio-emitting beams that escape upward along open magnetic field lines. If the beam of electrons travels along a closed loop, the plasma

\footnotetext{
* The movie is available in electronic form at http://www . aanda.org
}

density gradient, and thus the frequency drift, reverses, yielding J-bursts of U-bursts (e.g., Labrum \& Stewart 1970). N-bursts (Caroubalos et al. 1987) are occasionally seen as well, when a second reversal of the gradient occurs due to magnetic mirroring in the loop footpoints.

Type III bursts have been observed from frequencies as high as $\sim 1 \mathrm{GHz}$ at the base of the corona to about $30 \mathrm{kHz}$ at $1 \mathrm{AU}$ and even lower farther out. In several cases, type III bursts from the corona continue into the interplanetary (IP) medium; these events are indicative of open field lines emanating from the vicinity of the acceleration region and extending into the IP medium. However, not every coronal type III burst, even if strong, produces an IP type III burst (e.g., Gopalswamy et al. 1998). This may be due to the complete energy loss of the electrons.

The frequency drift rate of type III bursts can be directly converted into a drift of ambient coronal density with height, and the electron beam speed can be computed if a coronal density model is provided. The speeds derived range from about $c / 3$ in the low corona to about $c / 10$ at $1 \mathrm{AU}$.

The properties of type III bursts have been used for the diagnosis of the electron acceleration region. Aschwanden \& Benz (1997) inferred the ambient coronal density of the acceleration region from the plasma frequency at the separatrix between correlated type III bursts and reverse-slope bursts. Their results indicated that electrons are accelerated above the soft X-ray flaring loop-tops. More recently, this result was refined by Reid et al. $(2011,2014)$, who exploited the connection between the 
starting frequency of type III bursts and the HXR spectral index and found that the height of the acceleration region varied between 25 to $200 \mathrm{Mm}$.

Type III bursts usually come from active regions (e.g., Saint-Hilaire et al. 2013) and typically appear in groups of some ten or more bursts. Very many flares, particularly impulsive flares (e.g., Cane \& Reames 1988), exhibit type III bursts at their onset. Several publications are available about the statistical relationship between type III bursts and HXR bursts (e.g., Kane 1972, 1981; Hamilton et al. 1990; Aschwanden et al. 1995). More recently, Benz et al. $(2005,2007)$ studied a large number of flares above GOES class C5 and found that nearly all flares were associated with coherent radio emission between $4 \mathrm{GHz}$ to $100 \mathrm{MHz}$; most of their flares with coherent radio emission had type III bursts.

Type III radio bursts are known to be associated not only with flares, but also with $\mathrm{H} \alpha$ ejecta (Axisa et al. 1973), X-ray bright points (Kundu et al. 1994, 1995a), soft X-ray transient brightenings (Nindos et al. 1999), and soft X-ray or/and extreme-UV (EUV) jets (Kundu et al. 1995b; Raulin et al. 1996; Pick et al. 2006; Nitta et al. 2008; Innes et al. 2011; Krucker et al. 2011; Klassen et al. 2011, 2012; Chen et al. 2013a,b). Jets that are correlated with type III bursts accompany flaring activity that ranges from weak transient brightenings and subflares (Kundu et al. 1995b; Raulin et al. 1996; Nitta et al. 2008) to M-class flares (Krucker et al. 2011; Chen et al. 2013a). Their dimensions may range from about $10^{4} \mathrm{~km}$ to more than about $10^{5} \mathrm{~km}$. They appear most frequently at the edge of active regions and sometimes (Pick et al. 2006) close to the border of an active region and a coronal hole.

The correlation of type III bursts with soft X-ray/EUV jets and weak transient brightenings indicates that particle acceleration may take place when jets and weak transient brightenings occur. This is also supported by the in situ detection of electron spikes at energies below $300 \mathrm{keV}$ (Klassen et al. 2011, 2012), whose timing is well correlated with type III bursts and EUV jets. For jet events correlated with both type III bursts and electron events observed in situ, the flare geometry can be explained by reconnection between open and closed magnetic field lines (the so-called interchange reconnection; Krucker et al. 2011). The previously open field lines form the flaring loops, whereas heating along the newly opened field lines is weaker because material can easily be lost due to the open geometry of the field. Type-III-producing electrons escape along the newly opened field line.

In this article we analyze three tiny events detected by the Nançay Radio Heliograph (NRH). Two of the events were associated with type III bursts that were traced from the corona into the IP medium. These events are remarkable because they show that strong IP type III bursts may originate in electrons released in very weak events, provided that the electrons have access to open field lines. The article is organized as follows: in Sect. 2 we describe the observations and present the results, while in Sect. 3 we discuss the conclusions.

\section{Observations and results}

Figure 1 gives a composite dynamic spectrum in the range $700 \mathrm{MHz}$ to $0.02 \mathrm{kHz}$, together with 1-dimensional images (EW $\&$ NS) from the NRH. In the time interval of $2 \mathrm{~h}$ shown in the figure, three weak emissions are seen in the NRH 1D images; out of these, events B and C were accompanied by interplanetary type IIIs, while A was not. We note that apart from these three weak emissions, the Sun was very quiet at metric wavelengths, with the quiet Sun clearly visible in the NRH 1D images.

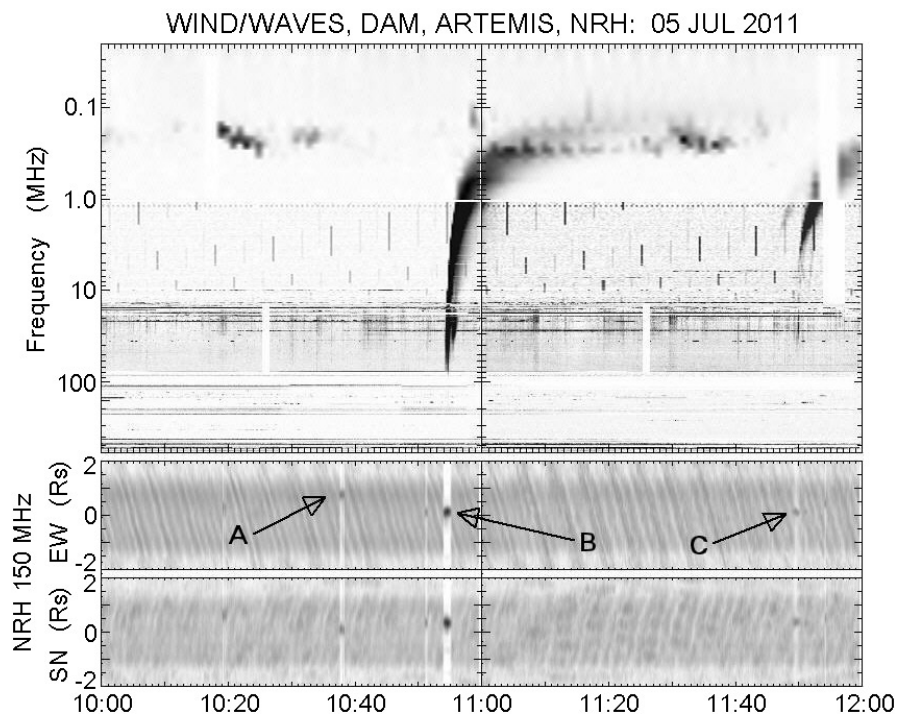

Fig. 1. Composite dynamic spectrum and NRH 1D images (from the Radio Monitoring site $^{1}$ ).

\subsection{EUV and X-ray data for event $B$}

Based on the NRH positions, we identified in SDO/AIA images a small flaring loop, $\sim 20^{\prime \prime}$ long, located at $3.2 \mathrm{~W}, 16.4 \mathrm{~N}$, as the source of the strongest event, B (Fig. 2). The HMI continuum image (top row) shows a group of small pores, and the magnetogram shows two negative-polarity patches surrounded by a positive-polarity magnetic field. Before the event (left column) there are two sets of loops, joining each negative patch with the nearby positive polarities.

There was no detectable emission in the $1700 \AA$ band. In all other AIA wavelength bands the event started with brightenings at the footpoints located in opposite-polarity regions within the western set of pre-existing loops; this was followed by emission along the entire loop in all bands except at 1600 and $304 \AA$. The overall structure of the emission is well visible in the intensity RMS images, shown in the rightmost column of Fig. 2; here we can see that in the high-temperature channels the loop consists of at least two distinct strands, which are not parallel to each other, but their projected distance varies from $\sim 3.5^{\prime \prime}$ near the middle of the loop to $\sim 5.5^{\prime \prime}$ at the footpoints. The footpoints themselves consist of at least two components.

No emission associated with the event was detected in the GOES flux data in the 1 to $8 \AA$ band above the noise level of $4 \times 10^{-9} \mathrm{Wm}^{-2}$, while the total flux of the Sun in the 0.5 to $4 \AA$ band was below the detection limit of $10^{-9} \mathrm{Wm}^{-2}$. We also found no trace of the event in the RSTN microwave patrol data. RHESSI was in the Earth shadow at the time, thus we have no hard X-ray information. Hinode was not observing the region at the time of the events, while GOES/SXI images show the two sets of loops mentioned above as bright points, and our event is barely discernible as a very weak brightening of the western bright point. The SXI images show that the flaring loop, located near the central meridian, was close to the border of a small coronal hole that was also visible in the NRH quiet-Sun image (Fig. 3).

Time profiles of the event in the $1600 \AA$ and the EUV bands of AIA, integrated in the direction perpendicular to the loop, are presented in Fig. 4. Their most important aspect is that the flux profiles of the entire event (left column in the figure) show, in

\footnotetext{
1 http://secchirh.obspm.fr/index.php
} 


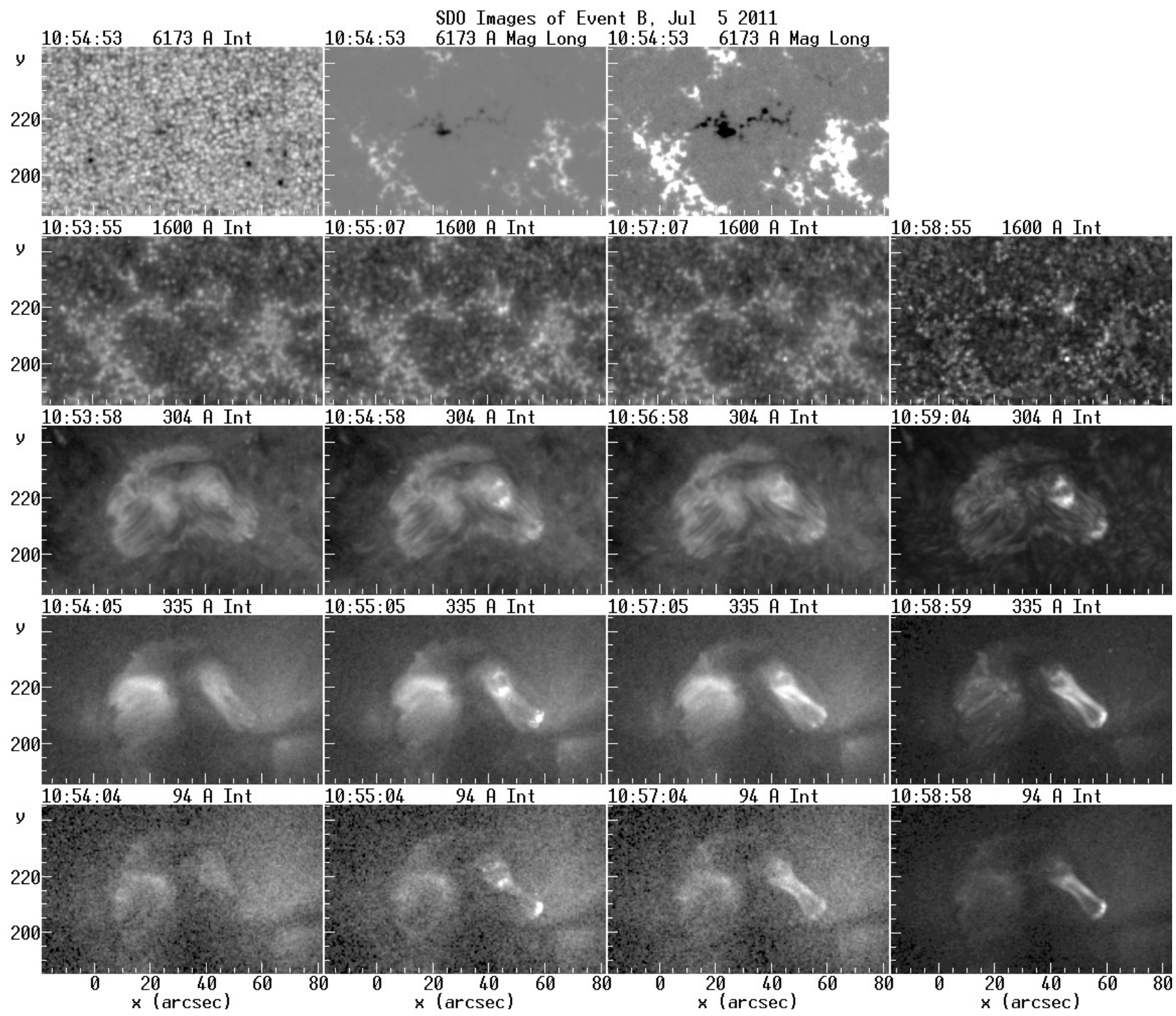

Fig. 2. SDO images of the flaring loop associated with event $B$. The top row shows an HMI white-light image with the corresponding magnetogram shown both with the full intensity range $( \pm 975 \mathrm{G})$ and saturated at $\pm 200 \mathrm{G}$. The other rows show AIA images in the $1600,304,335$ and $94 \AA$ Aands. The right-most column gives the RMS of the intensity during the event at the corresponding band. In this and all figures, the contrast for each wavelength band has been adjusted for best viewing.

addition to an impulsive component, a post-burst increase, most prominent in the 193, 211, 335, and $94 \AA$ channels.

As evidenced by the time profiles of the loop (second column in Fig. 4), the post-burst is associated with the body of the loop, while the time profiles of the footpoints (third and fourth column in the figure) were impulsive; there is some post-burst emission from the NE footpoint, but this is, most probably, due to the fact that this footpoint cannot be fully separated from the loop body due to projection effects. The emission from the body of the loop first appeared in the hot AIA band of $94 \AA$ and gradually in the cooler 335, 211, and 193 bands. This behavior is similar to that of two weak events described by Alissandrakis \& Patsourakos (2013), which had one footpoint inside a sunspot umbra and loop lengths of 44 to $53^{\prime \prime}$; they were interpreted in terms of coronal loop cooling, following an impulsive heating to multimillion $\mathrm{K}$ temperatures, with the heating phase being unobservable due to the low emission measure associated with it.
Although a detailed analysis of the event in the EUV is beyond the scope of this article, we would like to report here some interesting results derived from a differential emission measure (DEM) analysis of the AIA images, which we performed using the algorithm of Plowman et al. (2013). This is the same algorithm as was used by Alissandrakis \& Patsourakos (2013) and by Alissandrakis et al. (2013). We obtained a satisfactory fit to the data for most of the field of view, with a somewhat less satisfactory fit at the footpoints. Figure 5 shows selected DEM images in four temperature ranges $\left(1-2 \times 10^{6} \mathrm{~K}, 2-4 \times 10^{6} \mathrm{~K}, 4-8 \times 10^{6} \mathrm{~K}\right.$, and above $\left.8 \times 10^{6} \mathrm{~K}\right)$, together with HMI magnetic field and intensity images. A time sequence of DEM images and differences is shown in the movie attached to Fig. 5.

Before the event, a system of loops was visible at temperatures between 2 and $4 \times 10^{6} \mathrm{~K}$, spanning opposite magnetic polarities, with the rightmost end of the loops being near a small pore. The first brightenings appeared at 10:54:12 UT at 

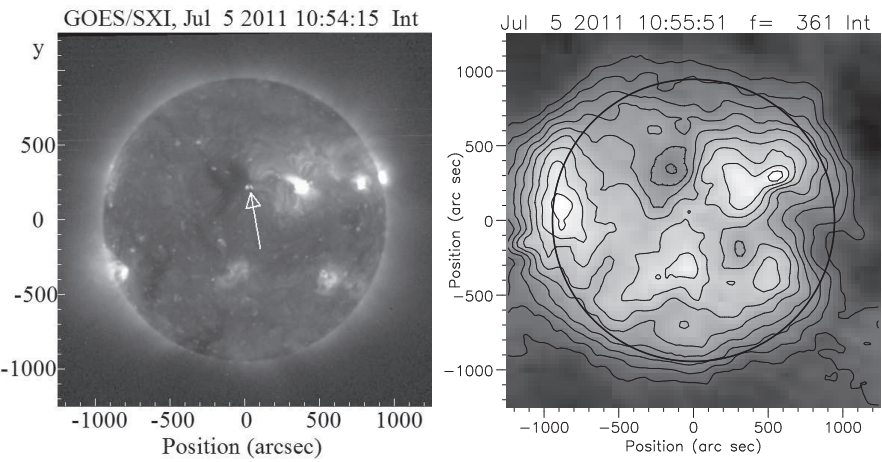

Fig. 3. Full disk images of the quiet sun: SXI, TM filter (left) and NRH, $361 \mathrm{MHz}$ (right, contours from $10^{5}$ to $9 \times 10^{5} \mathrm{~K}$ in steps of $10^{5} \mathrm{~K}$ ). The arrow in the SXI image points to the two pre-flare bright points
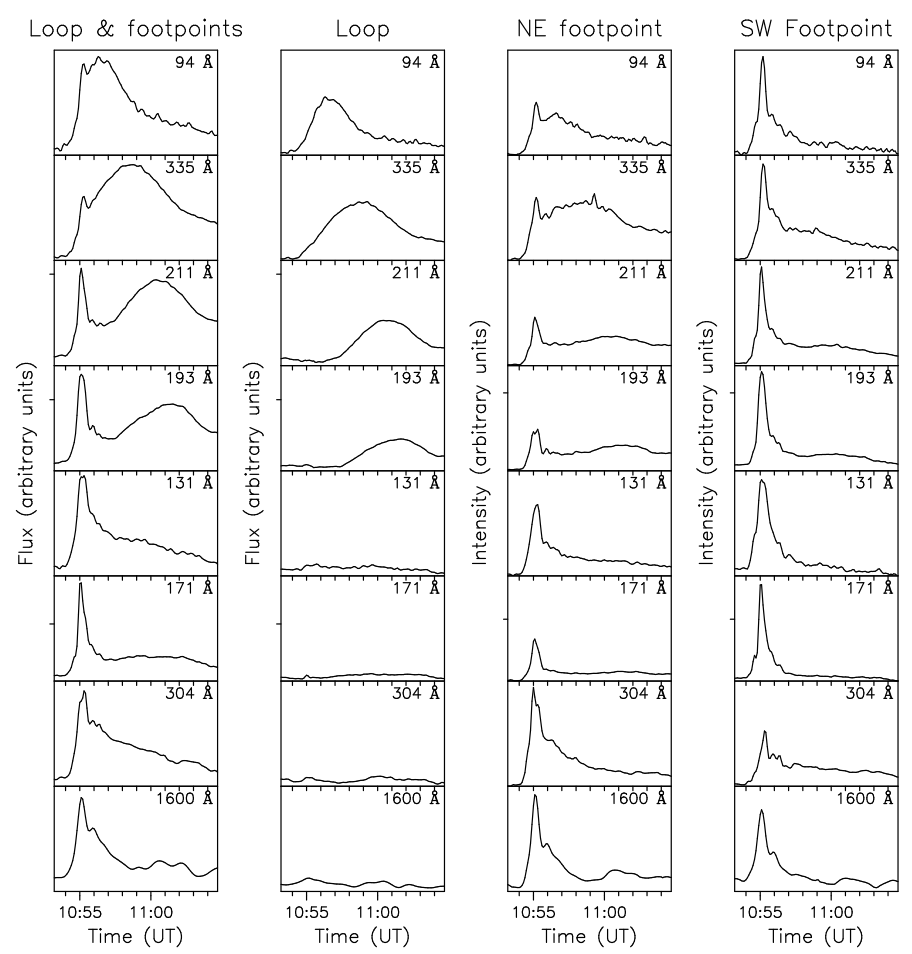

Fig. 4. Flux of the entire event B, the loop and the footpoints, as a function of time for the AIA wavebands.

locations $\mathbf{b}$ and $\mathbf{c}$ (arrows in Fig. 5). We note that although location $\mathbf{c}$ was near the rightmost edge of the pre-existing loops, location b was not near the leftmost edge (location a in Fig. 5), but was instead associated with a weak negative-polarity patch in between; brightenings at location a appeared $\sim 24$ s later, at 10:54:36 UT.

Loop emission first appeared at $T>8 \times 10^{6}$ near the upper footpoint at location $\mathbf{b}$ and quickly spread to the upper footpoint at location $\mathbf{c}$ with a projected velocity (hence a lower limit to the true velocity) of $\sim 400 \mathrm{~km} \mathrm{~s}^{-1}$, becoming stronger at lower temperatures, while withering away at high temperatures. A second loop appeared around 10:56:26 near the lower footpoint at location $\mathbf{b}$ and spread toward the lower footpoint at location $\mathbf{c}$ with an apparent velocity of $\sim 80 \mathrm{~km} \mathrm{~s}^{-1}$ in the $4 \times 10^{6} \mathrm{~K}<T<8 \times 10^{6} \mathrm{~K}$ range. Later in the event, the emission of the upper loop extended to footpoint a. We note that the footpoint emission had weakened considerably by the time the flaring loops had fully developed. We also note that both flaring loops were inclined by about $10^{\circ}$ with respect to the pre-flare loops.
The overall picture is that the first evidence of energy release was the heating of the footpoints to temperatures of $\sim 10^{7} \mathrm{~K}$; this was followed by the loop emission, first appearing at high temperatures and cooling gradually. The apparent expansion of the flaring loops from footpoint $\mathbf{b}$ to footpoint $\mathbf{c}$ is suggestive of evaporation of material heated in the course of the energy release process (cf. Fisher et al. 1984).

\subsection{Radio observations}

Returning to the metric data, we show in Fig. 6 dynamic spectra of the event in the metric-decametric range from the ARTEMIS sweep frequency receiver (ASG) and from the Bleien spectrograph. Unfortunately, the high-sensitivity, high-cadence acoustico-optic receiver of ARTEMIS (SAO) was not operating.

In the decametric range the dynamic spectrum shows two groups of type III bursts, followed by a type V burst. In the metric range the type III emission is weak and is detectable up to $200 \mathrm{MHz}$ in the ARTEMIS spectrum and up to about $300 \mathrm{MHz}$ in the Bleien spectrum. The principal type III group shows a fairly complex structure with several components, apparently corresponding to individual electron beams. This complex structure is more apparent in the much more sensitive NRH data, and the brightness temperature as a function of time is shown in Fig. 7, left panel. The maximum is at $150.9 \mathrm{MHz}\left(2.7 \times 10^{9} \mathrm{~K}\right)$ and the weakest peak is at $408 \mathrm{MHz}\left(9.4 \times 10^{6} \mathrm{~K}\right)$. We note the great decrease in brightness, by a factor of about 300, between low and high frequencies; in particular, there is a brightness drop by more than a factor of 10 between 228 and $270.8 \mathrm{MHz}$ that makes the time profiles look different. We also note that the maximum brightness temperature of type III bursts is reported to range from $10 \mathrm{MK}$ up to about $10^{12} \mathrm{~K}$ for coronal type III bursts (e.g., Saint-Hilaire et al. 2013) and up to about $10^{15} \mathrm{~K}$ in interplanetary type III bursts (e.g., Melrose 1989).

Compared to the EUV evolution (Fig. 7, right panel), the principal beams appeared during the rise phase of the impulsive component of the EUV emission, with some other beams, seen best at 270.7 and $327 \mathrm{MHz}$, extending slightly beyond the maximum.

The dynamic spectra of events B and C, as recorded by the WAVES instruments on STEREO A, WIND and STEREO B are given in Fig. 8. WIND/WAVES (Bougeret et al. 1995) has a thermal noise receiver (TNR), operating in the $4-256 \mathrm{kHz}$ range, and two radio band receivers, RAD1 operating from 20 to $1040 \mathrm{kHz}$ and RAD2 from 1.075 to $13.825 \mathrm{MHz}$; the low-frequency receiver (LFR) of STEREO/WAVES (Bougeret et al. 2008) operates in the 2.5 to $160 \mathrm{kHz}$ range, and the two high-frequency receivers operate from 125 to $1975 \mathrm{kHz}$ (HFR1) and from 2025 $\mathrm{kHz}$ to $16.025 \mathrm{MHz}$. At the time of our events, STEREO A was $97.6^{\circ}$ ahead of the Earth and STEREO B was $92.5^{\circ}$ behind.

Both bursts are visible in all three dynamic spectra. Event B is detectable down to about $100 \mathrm{kHz}$ in the WIND spectrum. Assuming that the electron density is inversely proportional to the square of the distance, which is valid for large distances (Leblanc et al. 1998) and under conditions of constant solar wind speed, the frequency is related to the distance from the Sun, $r$, as

$r=r_{\mathrm{E}} s f_{\mathrm{p}, \mathrm{E}} f^{-1}$,

where $r_{\mathrm{E}}$ is the distance to Earth, $s$ is 1 for the emission at the plasma frequency and 2 for harmonic emission, $f$ is the frequency of observation and $f_{\mathrm{p}, \mathrm{E}}$ is the plasma frequency of the solar wind near the Earth. The latter can be measured from the TNR data (horizontal ridge in Fig. 8), and its value was 


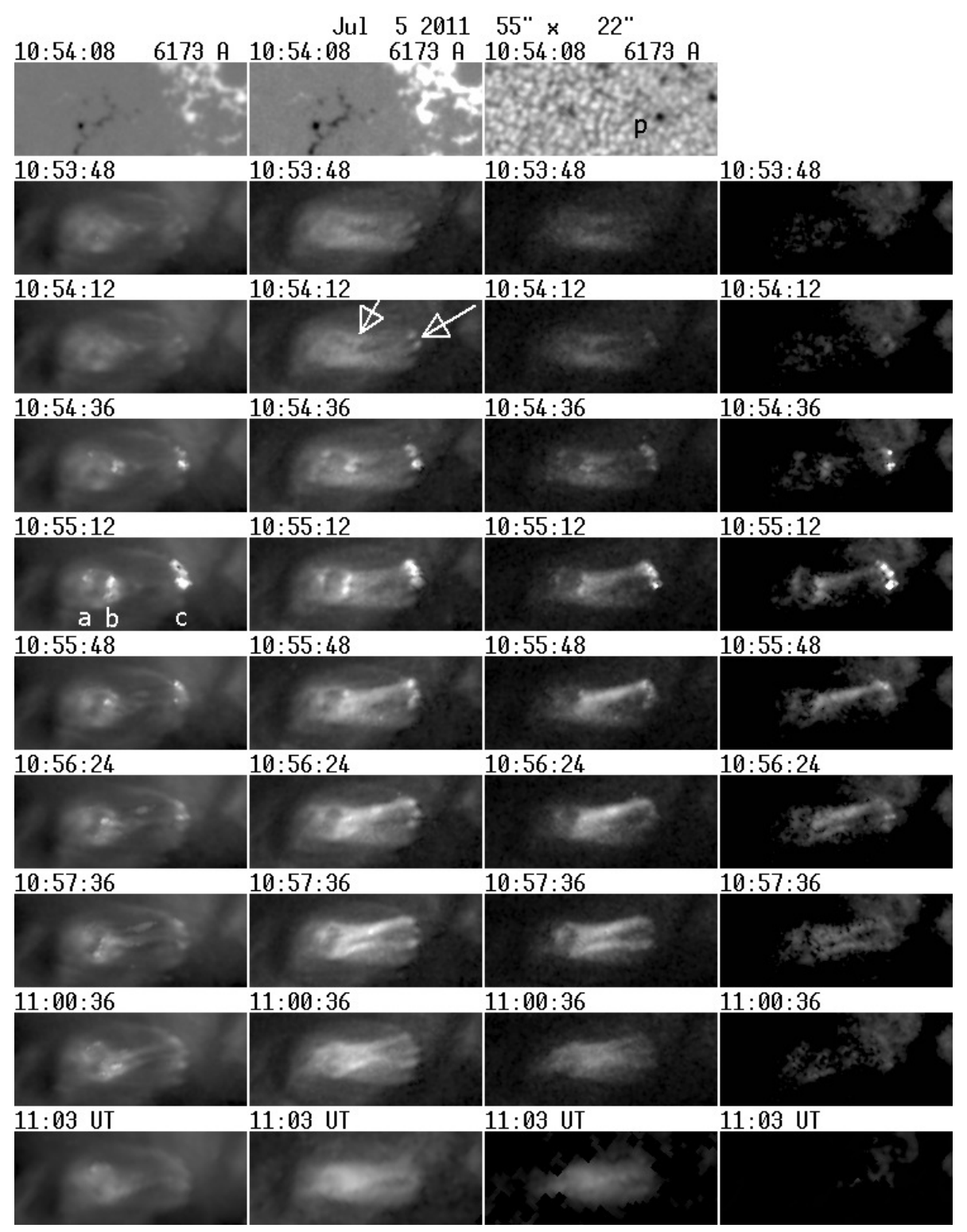

Fig. 5. Selected images of the DEM in the temperature ranges $1-2 \times 10^{6} \mathrm{~K}$ (first column, maximum value $\left.2.5 \times 10^{28} \mathrm{~cm}^{-5}\right), 2-4 \times 10^{6} \mathrm{~K}(\mathrm{sec}$ ond column, maximum value $\left.3.0 \times 10^{28} \mathrm{~cm}^{-5}\right)$, $4-8 \times 10^{6} \mathrm{~K}$ (third column, maximum value $2.0 \times 10^{28} \mathrm{~cm}^{-5}$ ) and above $8 \times 10^{6} \mathrm{~K}$ (right column, maximum value $\left.2.0 \times 10^{28} \mathrm{~cm}^{-5}\right)$ in the course of event B. The contrast of the display ( 0.6 for the first column and 0.4 for the others) was adjusted for best viewing. The top row shows HMI magnetograms (full range and saturated at $\pm 300 \mathrm{G}$ ) and a while-light image. The arrows in the 10:54:12 UT image mark the first brightenings, $\mathrm{a}, \mathrm{b}$ and $\mathrm{c}$ in the 10:55:12 UT image mark three sets of footpoints; $p$ in the white-light image marks a small pore. The field of view is 55 by $22^{\prime \prime}$ and the images have been rotated for easier viewing. A movie available online shows the full time sequence of DEM images (top row of movie) as well as DEM difference images (bottom row of the movie).

$21 \mathrm{kHz}$ in our case. Hence the $100 \mathrm{kHz}$ level is at $r \sim 45 R_{\odot}$ for $s=1\left(n_{\mathrm{e}}=180 \mathrm{~cm}^{-3}\right)$ and $\sim 90 R_{\odot}$ for harmonic emission $\left(n_{\mathrm{e}}=45 \mathrm{~cm}^{-3}\right)$ In SWAVES-A the burst is detectable down to $\sim 250 \mathrm{kHz}$ and in SWAVES-B down to $\sim 120 \mathrm{kHz}$, the differences between them and with WIND/WAVES being due to directivity and/or sensitivity effects.

We computed the average beam velocity from the variation of the time of the strongest emission as a function of frequency. We assumed that the electrons moved along a Parker spiral, rooted near the flaring loop, and that the magnetic field became radial at $3 R_{\odot}$; we used Eq. (1) to associate the frequency with the distance from the Sun, we adopted a solar wind speed of $450 \mathrm{~km} \mathrm{~s}^{-1}$ and took into account the light travel-time. Refraction effects were ignored in this computation; they would be stronger for emission in the fundamental.

The fit was better for emission at the harmonic of the plasma frequency and, combining the data from all three satellites, we obtained a value of $41 \mathrm{Mm} \mathrm{s}^{-1}$ for the beam velocity along the magnetic field lines of force at $3 R_{\odot}$ and a deceleration of $-15 \mathrm{~km} \mathrm{~s}^{-2}$, with estimated uncertainty of $1 \mathrm{Mm} \mathrm{s}^{-1}$ and $1 \mathrm{~km} \mathrm{~s}^{-2}$, respectively; thus the velocity dropped to $39 \mathrm{Mm} \mathrm{s}^{-1}$ at $10 R_{\odot}$ and to $25 \mathrm{Mm} \mathrm{s}^{-1}$ at $50 R_{\odot}$. Fitting the data of each individual satellite, we obtained a velocity range of $39-41 \mathrm{Mm} \mathrm{s}^{-1}$ and deceleration in the range of -16 to $-27 \mathrm{~km} \mathrm{~s}^{-2}$. If, instead, the emission was at the fundamental, the corresponding values of the velocity and the deceleration are $19 \mathrm{Mm} \mathrm{s}^{-1}$ and $-7 \mathrm{~km} \mathrm{~s}^{-2}$.

This means that the electron beam associated with our event had a moderate speed, $\sim 0.14 \mathrm{c}$. This result is consistent with previous studies: derived speeds for type III bursts can range from $>0.5 c$ in the corona (Poquérusse 1994; Klassen et al. 2003) down to about $0.1 c$ near the Earth (e.g., Dulk et al. 1987). It agrees very well with two particular statistical studies of the electron beam speeds inferred from decimetric type III bursts (Aschwanden et al. 1995; Meléndez et al. 1999).

For the emission flux, we made an estimate at $1 \mathrm{MHz}$ by comparing our data with those of two bursts shown in Figs. 3 and 4 of Krupar et al. (2014). We obtained flux values of about $4 \times 10^{-18} \mathrm{Wm}^{-2} \mathrm{~Hz}^{-1}$ and $0.9 \times 10^{-18} \mathrm{Wm}^{-2} \mathrm{~Hz}^{-1}$ for SWAVES $\mathrm{A}$ and $\mathrm{B}$, respectively; compared to the flux distribution derived from 152 bursts by Krupar et al. (2014), our event is near the average, which at $1 \mathrm{MHz}$ is about $2.5 \times 10^{-18} \mathrm{Wm}^{-2} \mathrm{~Hz}^{-1}$. 

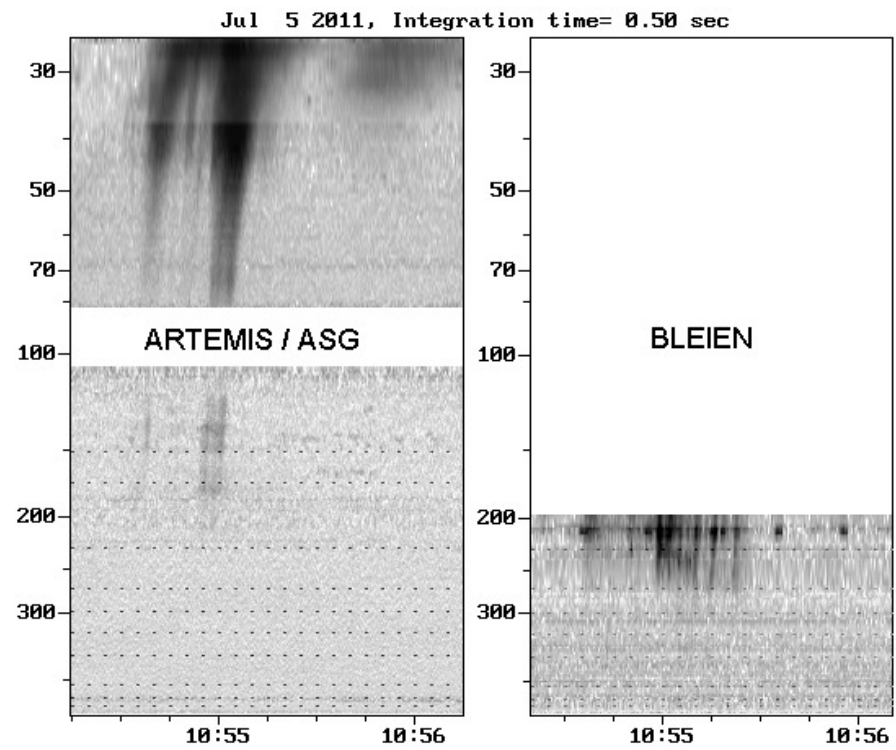

Fig. 6. Dynamic spectra from ARTEMIS-IV and Bleien. Dotted lines mark the frequencies of the NRH. The Bleien data are from the Callisto site $^{2}$.

\subsection{Burst positions in the metric range}

We computed 2D maps from the NRH visibilities. For this, it was necessary to introduce phase corrections to the visibilities, which were derived by self-calibration, using strong point-like sources. In all frequencies the sources had a simple structure; they were slightly resolved, and their size decreased with frequency (Fig. 9).

From the position of the emission peaks at each frequency we constructed 2D histograms of the number of peaks as a function of $x$ and $y$ position and summed all frequencies. The result is shown in the left panel of Fig. 10, together with an image of the loop; also shown in the figure is a magnetogram and an SDO image at $211 \AA$ with superimposed magnetic field lines. We first note that there is a net drift of positions from high to low frequencies, apparently reflecting the average upward motion of the electron beams along the magnetic field. The fact that we are near the coronal hole boundary explains why the beams found open magnetic field lines to escape into the interplanetary space.

The positions at $150.9,173.2$, and $228 \mathrm{MHz}$ are spread over elongated regions $10^{\prime \prime}$ wide and $70^{\prime \prime}$ long; although the extent of the regions is smaller than the instrumental beam (cf. Fig. 9), this displacement is most probably real both because it is not a random spread (it is much longer than it is wide) and, most important, there is a systematic drift from NW to SE during the 2 min of the radio data. Thus the observed drift apparently reflects the fact that we have a multitude of individual beams, each following a slightly different field line.

We note that there is a net displacement of the radio sources from the flaring loop, which amounts to about 55" for high frequencies. As the radio sources were located in a region of high density gradient, between the coronal hole and the nearby closed magnetic configurations, their observed position may have been affected by refraction. It is beyond the scope of this article to model these effects in detail, but we still did some indicative ray-tracing computations using simple models described by

\footnotetext{
2 http://soleil.i4ds.ch/solarradio/callistoQuicklooks/
}

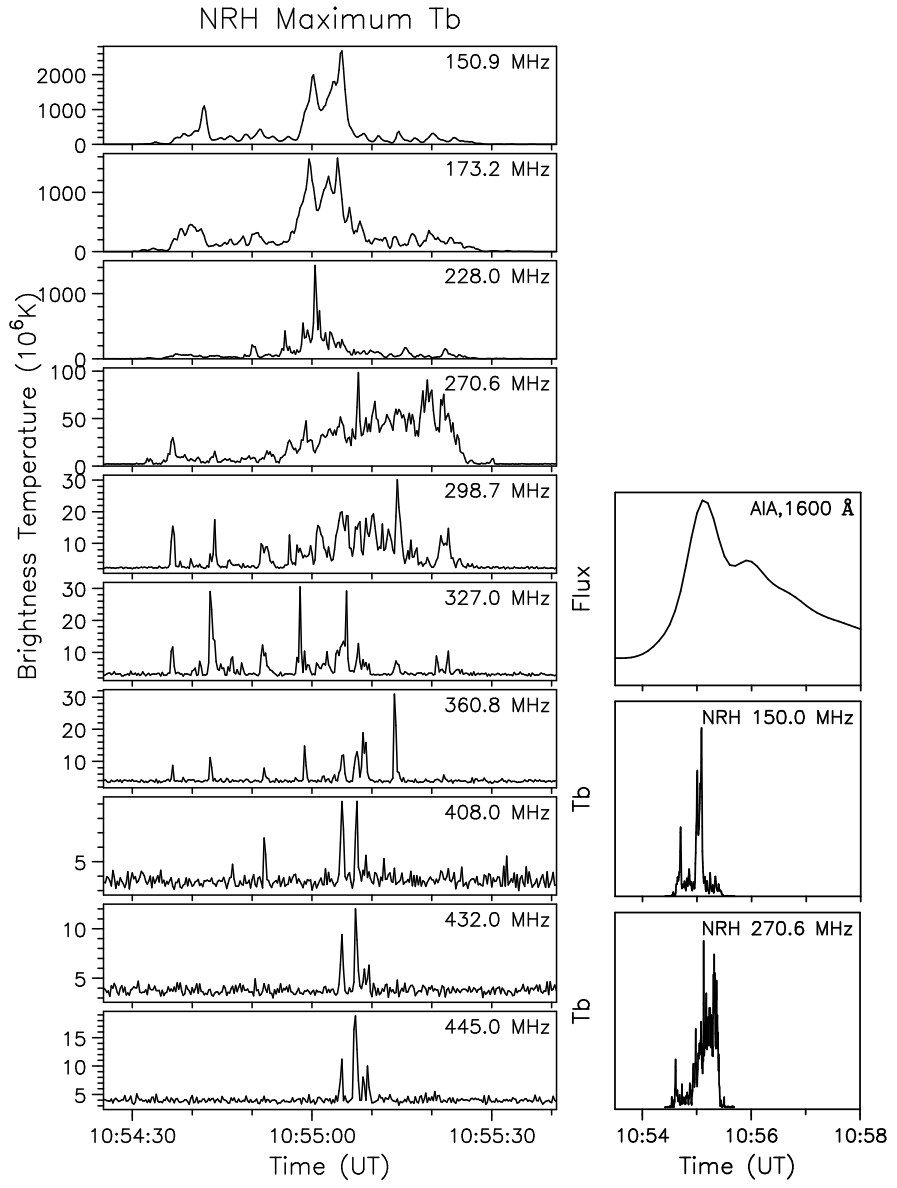

Fig. 7. Maximum brightness temperature as a function of time; note that the scales are different for each frequency (left). Comparison of the 270.6 and 150 light curves with that of the $1600 \AA$ A AIA band (right).

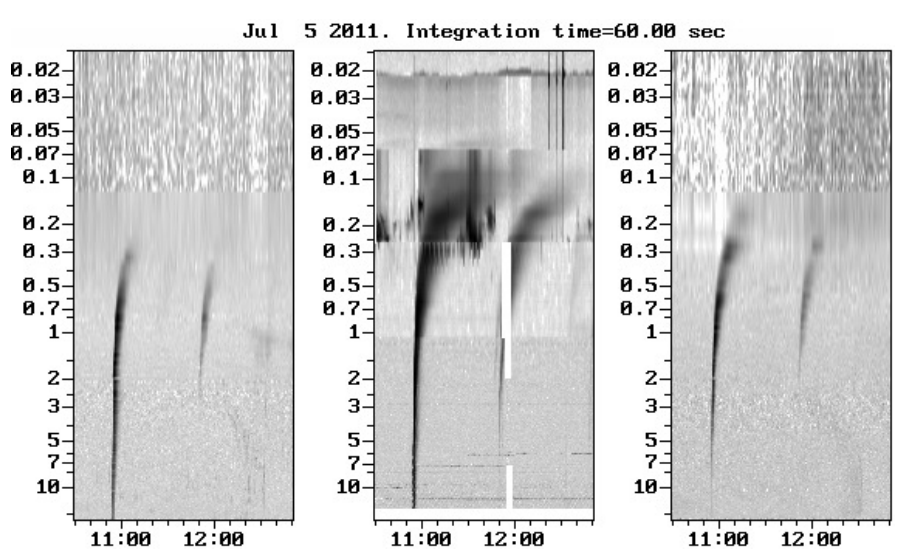

Fig. 8. Dynamic spectra of events B and C from SWAVES-A (left panel), WIND/WAVES (middle panel) and SWAVES-B (right panel). The frequency is in MHz.

Alissandrakis (1994), assuming emission at the harmonic of the plasma frequency.

We found that, as a result of refraction, the true position of the radio source is closer to the center of the coronal hole than the observed position; hence the true distance of the radio sources from the flaring loop is even greater than the observed distance, and this effect will be stronger if the emission is at the 


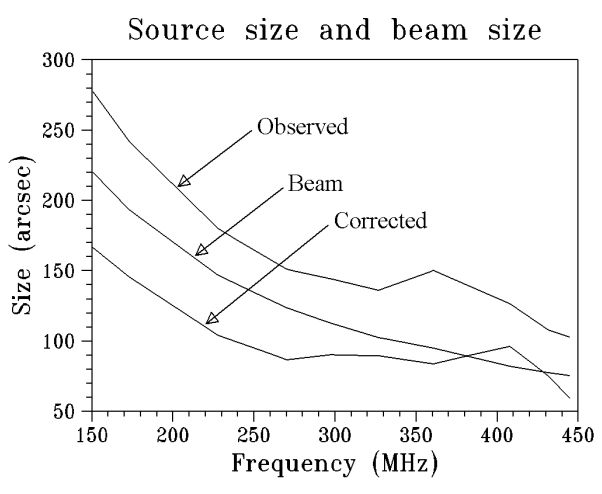

Fig. 9. Observed source size, beam size, and corrected source size.

NRH burst positions, B_long \& PFSS lines; Jul $52011300^{\prime \prime}$ x 300" 10:55:02 Int 10:58:38 6173 A Mag 16:34:14 211 A Int

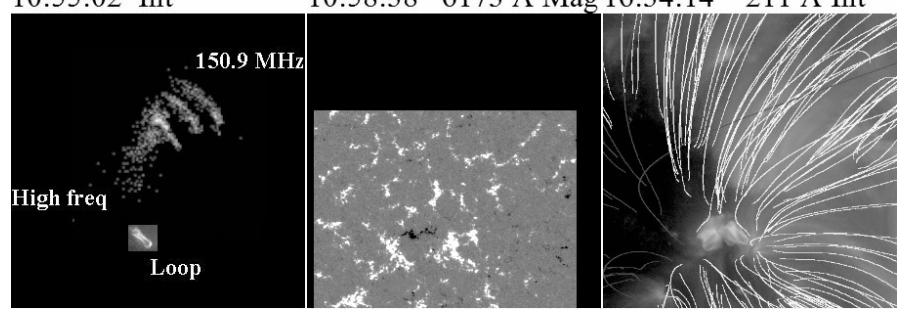

Fig. 10. From left to right: combined $2 \mathrm{D}$ histograms of the position of $\mathrm{NRH}$ sources at all frequencies; the inset shows an image of the loop at $335 \AA$; HMI/SDO magnetogram; lines of force of the potential magnetic field extrapolation superimposed on a $211 \AA$ AIA image ${ }^{3}$.

fundamental. The computed displacement is $\sim 10^{\prime \prime}$ at $400 \mathrm{MHz}$ and increases to $\sim 30^{\prime \prime}$ at $160 \mathrm{MHz}$, an effect that will cause the true orientation of the magnetic field lines traced by the beam to be less inclined than suggested in Fig. 10 and closer to the direction of the potential field lines.

The velocity of the beam can be estimated from the frequency drift of the type III emission. We obtained consistent values of the drift, $\mathrm{d} \ln f / \mathrm{d} t=-0.25 \mathrm{~s}^{-1}$, both from the ARTEMIS spectra and the NRH data around $160 \mathrm{MHz}$. We note that

$v \cos \theta=-2 H \frac{\mathrm{d} \ln f}{\mathrm{~d} t}$,

where $H$ is the density scale height, $v$ the velocity along the field lines, and $\theta$ the inclination of the beam trajectory with respect to the vertical (radial direction). To make the observed drift compatible with the velocity of $41 \mathrm{Mm} \mathrm{s}^{-1}$ estimated from the WAVES spectra (Sect. 2.2), a scale height of

$$
\frac{H}{\cos \theta}=83 \mathrm{Mm}=0.119 R_{\odot}
$$

is required.

Additional information can be obtained from the transverse velocity of the beam, $v_{\mathrm{t}}$, which can be computed from the displacement of the radio images and the delay of the corresponding intensity peaks; ignoring refraction effects, our measurements at 150.9 and $173.2 \mathrm{MHz}$, where individual peaks are easy to identify (cf. Fig. 7), give

$v_{t}=v \sin \alpha=38 \mathrm{Mm} \mathrm{s}^{-1}$

\footnotetext{
3 From http://sdowww. lmsal. com/suntoday/
}

where $\alpha$ is the angle between the beam velocity and the line of sight. From the measured transverse velocity and the value of the beam velocity estimated in Sect. 2.2, we obtain $\alpha=66^{\circ}$ from the above relation. We note here that the beam velocity deduced from the WAVES spectra for emission at the plasma frequency $\left(19 \mathrm{Mm} \mathrm{s}^{-1}\right)$ is incompatible with the measured transverse velocity; hence we may safely conclude that the emission was at the harmonic.

Moreover, the orientation of the projection of the beam trajectory on the plane of the sky can be measured from the position of the type III burst at high and low frequencies (Fig. 10), again ignoring refraction effects. From this and the angle $\alpha$, we can compute the angle between the beam and the radial direction through a coordinate transform taking into account the heliocentric position of the loop. In this way, we obtain $\theta=52^{\circ}$, hence $H=51 \mathrm{Mm}=0.073 R_{\odot}$ from Eq. (3).

Furthermore, the scale height depends on the temperature and on the distance from the center of the Sun, $r$,

$H=0.0712 T_{6}\left(\frac{R_{\odot}}{r}\right)^{2} \quad\left(\right.$ in units of $\left.R_{\odot}\right)$,

where $T_{6}$ is the temperature in units of $10^{6} \mathrm{~K}$ and a molecular weight $\mu=0.61$ has been assumed. A lower limit of $r$ can be estimated from the projected distance between high- and lowfrequency sources (Fig. 10), which is $0.118 R_{\odot}$, and the angles $\alpha$ and $\theta$ computed above. This gives $r \simeq 1.0757 R_{\odot}$, which, substituted into Eq. (5), gives an ambient temperature of $1.2 \times 10^{6} \mathrm{~K}$ at the level of formation of the $160 \mathrm{MHz}$ emission, which corresponds to an electron density of $3.8 \times 10^{8} \mathrm{~cm}^{-3}$ for emission at the harmonic.

These computations show that we can derive self-consistent results from the combined information about the beam velocity from WAVES and ARTEMIS spectra and the NRH images. The derived temperature and emission height are both on the low side of the expected values, but this might be because refraction effects could not be taken into account. We note, however, that the derived electron density is not much different from that predicted from coronal models: at $1.08 R_{\odot}$ the density is $4.2 \times 10^{8} \mathrm{~cm}^{-3}$ according to the model of Newkirk (1961) and $1.91 \times 10^{8} \mathrm{~cm}^{-3}$ according to the equatorial model of Saito (1970). Furthermore, the scale height of the Newkirk model corresponds to a coronal temperature of $1.4 \times 10^{6} \mathrm{~K}$, while the observed brightness temperature of the quiet Sun is $\sim 1 . \times 10^{6} \mathrm{~K}$ (Fig. 3 ).

We note that the comparison of the electron beam velocity in the corona and the interplanetary medium was made under the assumption that, in both cases, the electron pitch angle has a narrow distribution along the direction of the magnetic field; however, this may not be valid in the solar wind, due to waveparticle interactions that could broaden the pitch angle distribution and reduce the measured beam velocity.

\subsection{Events $A$ and $C$}

For the other two events we restrict ourselves to some general comments without presenting a detailed analysis. Starting with event $\mathrm{C}$, we note that it was associated with a flaring of the same loop that produced event $\mathrm{B}$, hence the similarity of the two events in the WAVES dynamic spectra. This flaring was weaker, which explains the weaker type III emission in the decametric and the hectometric range.

The case of event A is of greater interest; it only appeared in the NRH data (Fig. 1) without any counterpart in the dynamic spectra, although it was no less intense than event $C$, which was 

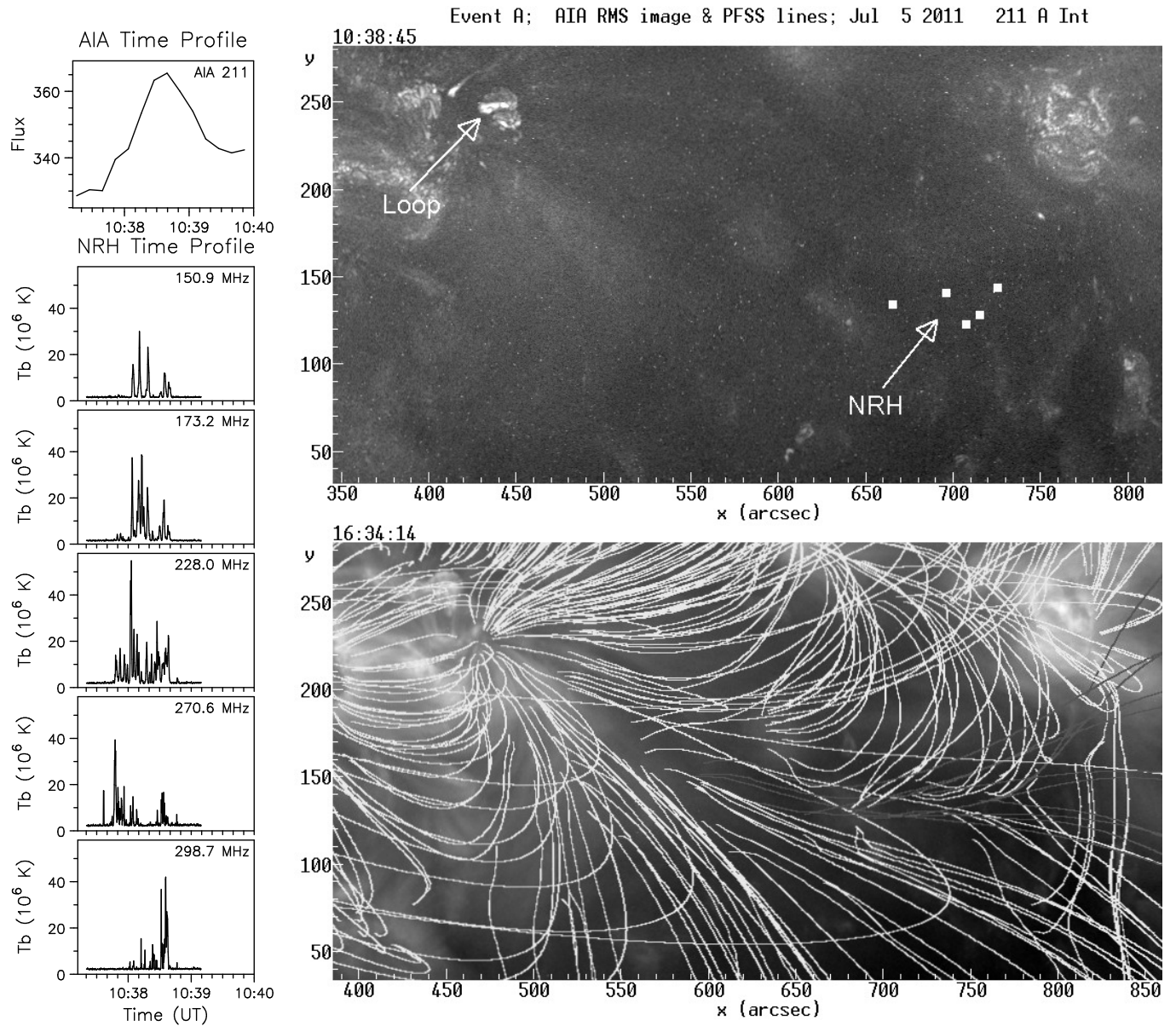

Fig. 11. Left: AIA and NRH time profiles of event A. Top right panel: image of the intensity RMS in the $211 \AA$ Aand; white squares mark the position of the NRH sources at (from E to W) 270.6, 298.7, 150.9, 173.2 and $228 \mathrm{MHz}$. Right, bottom: PFSS magnetic lines of force ${ }^{4}$.

associated with a weak type III burst. There were no brightenings in the immediate vicinity of the metric emission, but we were able to identify its origin on the basis of the time profiles: it was most probably associated with a small flaring loop located more than one-third of a solar radius away (Fig. 11). This loop, which was located about $450^{\prime \prime}$ west of the loop that produced events B and $\mathrm{C}$, is well visible in the image of the RMS variation of the intensity in the $211 \AA$ AIA band (Fig. 11, top right); we note that the jet seen to the east of the loop flared up a few minutes earlier than our event.

As in event $\mathrm{B}$, the metric emission consisted of many individual peaks during the rise phase of the EUV light curve (Fig. 11, left panel). Its brightness temperature was lower than that of event $\mathrm{B}$, and there was no detectable emission beyond 298.7 MHz. The metric sources did not show the regular arrangement of event B, but were rather scattered (Fig. 11, top right panel). Without dynamic spectra it is difficult to specify the nature of the emission, but some drifting peaks in the time profiles are indicative of electron beams moving upward. The geometry of the magnetic field, presented in the bottom right panel of Fig. 11, shows that this loop was embedded in a region of closed magnetic lines. Thus the closed magnetic field configuration explains why the electrons did not find their way into the interplanetary space.

\footnotetext{
${ }^{4}$ From http://sdowww. Imsal. com/suntoday/
}

\section{Discussion and conclusions}

Our basic conclusion is that strong type III bursts in the decametric and kilometric range may be produced by electrons released in very weak EUV events. Such events would have been missed in the EUV without the AIA superior cadence and resolution: our strongest event was not detectable in the GOES flux data and was barely detectable as a flaring bright point in GOES/SXI images. In all three cases, the associated events were tiny flaring loops; we did not find any evidence of any mass eruption in terms of a jet, micro-CME, or propagating disturbance.

The principal prerequisite for interpranetary type III emission is that the electron beams find open magnetic field lines to propagate in interplanetary space, as demonstrated by the fact that events $\mathrm{B}$ and $\mathrm{C}$, which originated near a coronal hole, caused interplanetary type III bursts, while event A, located inside a closed magnetic field region, did not. Furthermore, for event B, we found that the position of the metric sources, even at high frequencies, was displaced by about $55^{\prime \prime}$ with respect to the nearest footpoint of the associated flaring loop, the true distance being slightly larger due to refraction effects. A much larger distance of $\sim 300^{\prime \prime}$ separated the metric emission of event A from the associated loop. As the electrons are expected to be accelerated near the energy release site, the obvious question arises of how they got there.

The relatively large offset between the site of energy release and the metric type III burst loci presents a formidable challenge 
to models. It is well known that particle transport across the mean magnetic field can be dramatically enhanced (well above excursions of about the electron gyroradius in a collisional time) in the presence of turbulence because of the braiding of field lines owing to perpendicular magnetic fluctuations. However, the displacements we found between the metric sources and the flaring loops appear too large $\left(55-300^{\prime \prime}\right)$ to be interpreted in terms of cross-field transport of the energetic electrons. For example, Kontar et al. (2011) found that the cross-field transport of electrons with energies $8-25 \mathrm{keV}$ in a flaring loop yielded excursions that were not larger than $1-2^{\prime \prime}$.

The AIA data show no evidence for any small-scale CMElike event that could possibly act as driver for the displacement of the electrons producing the radio sources. Furthermore, it is unlikely that such large displacements were the consequence of systematic errors in NRH positional data, because care was taken to introduce phase corrections to the visibilities, which were derived by self-calibration. It is possible that the energetic electrons were transferred away from the flare site through ultrathin loop bundles (cf. Chen et al. 2013) that were below the AIA resolution and then gained access to open field lines via interchange reconnection. Clearly, more observations that will combine positional information of type III sources at several frequencies with high spatial and temporal resolution EUV observations are needed to check how often and under which circumstances displacements like the one reported here might possibly occur.

Even at this low intensity level, the metric emission consists of a multitude of sources, apparently associated with different electron beams, evidenced both from the time structure of the emission and from the displacement of the source position in the course of the event. Apparently, each beam is associated with a different episode of electron acceleration and, possibly, of energy release that all occur during the rise phase of the EUV emission (Fig. 7).

Using a simple model of the beam electrons propagating along a Parker spiral in interplanetary space and a density variation proportional to $r^{-2}$, we derived for event $\mathrm{B}$ the beam velocity $\left(41 \mathrm{Mm} \mathrm{s}^{-1}\right)$ and deceleration $\left(-15 \mathrm{~km} \mathrm{~s}^{-2}\right)$ from the observed time of maximum as a function of frequency in the three WAVES dynamic spectra. Combining this information with the drift rate measured from the metric dynamic spectra and the displacement of the radio sources with frequency and time measured from the $\mathrm{NRH}$ images, we obtained self consistent values for the height, the temperature, and the density at the level of formation of the $160 \mathrm{MHz}$ emission. We also found that the beam velocity computed under the assumption of emission at the plasma frequency was smaller than the measured transverse beam velocity, hence we conclude that the emission was at the harmonic.

Last but not least, we obtained interesting results from computing the differential emission measure from the AIA images. Our analysis for event B showed that the energy release occurred near the loop footpoints, which were heated to above $10^{7} \mathrm{~K}$ and cooled rapidly; the loop body was formed after the footpoint emission, again with a temperature exceeding $10^{7} \mathrm{~K}$, and cooled slowly.

Concluding this article, we would like to point out the importance of small events in understanding the energy release process. Although, as demonstrated here, such phenomena may turn out to be rather complex under high spatial and temporal resolution, they are still much simpler to study than large events and thus allow a more direct testing of theoretical models. They thus represent a very interesting class of phenomena for further study.
Acknowledgements. The authors thank the NRH staff for providing the original visibility data; particular thanks are due to Claude Mercier for his assistance with the self-calibration of the NRH visibilities. The authors gratefully acknowledge use of data from the SDO, Solar Monitor, Callisto and GOES/SXI data bases. The research of A.N. and S.P. has been partly co-financed by the European Union (European Social Fund -ESF) and Greek national funds through the Operational Program "Education and Lifelong Learning" of the National Strategic Reference Framework (NSRF) -Research Funding Program: "Thales. Investing in knowledge society through the European Social Fund". S.P. acknowledges support from an FP7 Marie Curie Grant (FP7-PEOPLE-2010-RG/268288).

\section{References}

Alissandrakis, C. E. 1994, Adv. Space Res., 14, 81

Alissandrakis, C. E., \& Patsourakos, S. 2013, A\&A, 556, A79

Aschwanden, M. J., \& Benz, A. O. 1997, ApJ, 480, 825

Aschwanden, M. J., Benz, A. O., Dennis, B. R., \& Schwartz, R. A. 1995, ApJ, 455,347

Alissandrakis, C. E., Kochanov, A. A., Patsourakos, S., et al. 2013, PASJ, 65, 8 Axisa, F., Martres, M. J., Pick, M., \& Soru-Escaut, I. 1973, Sol. Phys., 29, 163 Bastian, T. S., Benz, A. O., \& Gary, D. E. 1998, ARA\&A, 36, 131

Benz, A. O., Grigis, P. C., Csillaghy, A., \& Saint-Hilaire, P. 2005, Sol. Phys., 226, 121

Benz, A. O., Brajša, R., \& Magdalenić, J. 2007, Sol. Phys., 240, 263

Bougeret, J.-L., Kaiser, M. L., Kellogg, P. J., et al. 1995, Space Sci. Rev., 71, 231 Bougeret, J. L., Goetz, K., Kaiser, M. L., et al. 2008, Space Sci. Rev., 136, 487 Cane, H. V., \& Reames, D. V. 1988, ApJ, 325, 895

Caroubalos, C., Poquerusse, M., Bougeret, J.-L., \& Crepel, R. 1987, ApJ, 319, 503

Chen, B., Bastian, T. S., White, S. M., et al. 2013a, ApJ, 763, L21

Chen, N., Ip, W.-H., \& Innes, D. 2013b, ApJ, 769, 96

Dulk, G. A. 1985, ARA\&A, 23, 169

Dulk, G. A., Steinberg, J. L., Hoang, S., \& Goldman, M. V. 1987, A\&A, 173, 366

Fisher, G. H., Canfield, R. C., \& McClymont, A. N. 1984, ApJ, 281, L79

Gopalswamy, N., Kaiser, M. L., Lepping, R. P., et al. 1998, JGR (Space), 103, 307

Hamilton, R. J., Petrosian, V., \& Benz, A. O. 1990, ApJ, 358, 644

Innes, D. E., Cameron, R. H., \& Solanki, S. K. 2011, A\&A, 531, L13

Kane, S. R. 1972, Sol. Phys., 27, 174

Kane, S. R. 1981, ApJ, 247, 1113

Klassen, A., Karlicky, M., \& Mann, G. 2003, A\&A, 410, 307

Klassen, A., Gómez-Herrero, R., \& Heber, B. 2011, Sol. Phys., 273, 413

Klassen, A., Gómez-Herrero, R., Heber, B.. et al. 2012, A\&A, 542, A28

Kontar, E. P., Hannah, I. G., \& Bian, N. H. 2011, ApJ, 730, L22

Krucker, S., Kontar, E. P., Christe, S., Glesener, L., \& Lin, R. P. 2011, ApJ, 742, 82

Krupar, V., Maksimovic, M., Santolik, O., et al. 2014, Sol. Phys., 289, 3121

Kundu, M. R., Strong, K. T., Pick, M., et al. 1994, ApJ, 427, 59

Kundu, M. R., Raulin, J. P., Pick, M., \& Strong, K. T. 1995a, ApJ, 444, 922

Kundu, M. R., Raulin, J. P. Nitta, N., et al. 1995b, ApJ, 447, L135

Labrum, N. R., \& Stewart, R. T. 1970, Proc. Astr. Soc. Austr., 1, 316

Leblanc, Y., Dulk, G. A., \& Bougeret, J.-L. 1998, Sol. Phys., 183, 165

Meléndez, J. L., Sawant, H. S., Fernandes, F. C. R., \& Benz, A. O. 1999, Sol. Phys., 187, 77

Melrose, D. B. 1980, Plasma astrophysics, Vols. 1 and 2 (New York: Gordon and Breach)

Melrose, D. B. 1989, Sol. Phys. 120, 369

Melrose, D. B. 2009, in IAU Symp. 257, eds. N. Gopalswamy, \& D. F. Webb, 305

Newkirk, G., Jr. 1961, ApJ, 133, 983

Nindos, A., Kundu, M. R., \& White, S. M. 1999, ApJ, 513, 983

Nindos, A., Aurass, H., Klein, K.-L., \& Trottet, G. 2008, Sol. Phys., 253, 3

Nitta, N.V., Mason, G. M., Wiedenbeck, M. E., et al. 2008, ApJ, 675, L125

Pick, M., Mason, G. M., Wang, Y.-M., Tan, C., \& Wang, L. 2006, ApJ, 648, 1247

Plowman, J., Kankelborg, C., \& Martens, P. 2013, ApJ, 771, 2

Poquérusse, M. 1994, A\&A, 286, 611

Raulin, J.-P., Kundu, M. R., Hudson, H. S., Nitta, N., \& Raoult, A. 1996, A\&A, 306, 299

Reid, H. A. S., \& Ratcliffe, H. 2014, RA\&A, 14, 773

Reid, H. A. S., Vilmer, N., \& Kontar, E. P. 2011, A\&A, 529, A66

Reid, H. A. S., Vilmer, N., \& Kontar, E. P. 2014, A\&A, 567, A85

Saito, K. 1970, Ann. Tokyo Astron. Obs. Ser., 12, 53

Saint-Hilaire, P., Vilmer, N., \& Kerdraon, A. 2013, ApJ, 762, 60

Tsytovich, V. N. 1995, Lecture on Non-linear Plasma Kinetics, eds. V. N. Tsytovich, \& D. ter Haar 